\title{
HARMONIC DIFFERENTIAL WITH PRESCRIBED SINGULARITIES
}

\author{
BY \\ MITSURU NAKAI( ${ }^{(1)}$
}

Introduction

1. Throughout this paper we denote by $R$ an open Riemann surface and by $R_{0}$ a relatively compact subdomain of $R$ with the relative boundary $\partial R_{0}$ consisting of a finite number of mutually disjoint closed analytic Jordan curves. The open set $R_{1}=R-\bar{R}_{0}$ can be considered to be a neighborhood of the ideal boundary $\beta$ of $R$. For the sake of simplicity, we denote by $\alpha$ the common relative boundary $\partial R_{0}=\partial R_{1}$ and we fix the orientation of $\alpha$ positively with respect to the domain $R_{0}$.

A harmonic differential $\sigma$ defined on $\bar{R}_{1}=R_{1} \cup \alpha$ is called a harmonic singularity at $\beta$ and in case $\int_{R_{1}} \sigma \wedge{ }^{*} \sigma<\infty$, we say that the singularity $\sigma$ at $\beta$ is removable. A harmonic differential $\lambda$ on $R$ is said to have the harmonic singularity $\sigma$ at $\beta$ if $\lambda-\sigma$ is a removable harmonic singularity at $\beta$. The purpose of this paper is to discuss the following

Problem A. Find a harmonic differential $\lambda$ on $R$ having a given harmonic singularity $\sigma$ at $\beta$.

It is known (Ahlfors-Sario [1, p. 300]) that Problem A is solvable if $\sigma$ and ${ }^{*} \sigma$ are the restrictions to $\bar{R}_{1}$ of some closed $C^{1}$-differentials on $R$. We shall prove that if $R \notin O_{G}$, then Problem A is always solvable, and if $R \in O_{G}$, then Problem $\mathrm{A}$ is solvable if and only if $\int_{\alpha} \sigma=\int_{\alpha} *_{\sigma}=0$ (Theorem 2).

2. In Problem A, we may assume without loss of generality that $\sigma$ is a $C^{1}$-differential on $R$ whose restriction to $\bar{R}_{1}$ gives a harmonic singularity at $\beta$. In fact, take a subdomain $R_{\sigma}$ of $R$ such that $\bar{R}_{\sigma} \subset R_{0}$ and $\sigma$ is harmonic on $R-\bar{R}_{\sigma}$. We find a $C^{1}$ function $\phi$ on $R$ such that $\phi=1$ on a neighborhood of $\bar{R}_{1}$ and $\phi=0$ on a neighborhood of $\bar{R}_{\sigma}$. Then $\phi \sigma$ can be considered to be a $C^{1}$-differential on $R$ and $\phi \sigma \mid \bar{R}_{1}=\sigma$.

Let $\Gamma=\Gamma(R)$ be the Hilbert space of all square integrable differentials on $R$ which is the completion of square integrable $C^{\infty}$-differentials on $R$ with respect to the inner product $\left(\omega_{1}, \omega_{2}\right)=\int \omega_{1} \wedge * \omega_{2}$. We denote by $\Gamma_{e 0}=\Gamma_{e 0}(R)$ the closure of $\Gamma_{e 0}^{\infty}=\Gamma_{e 0}^{\infty}(R)=\left\{d f ; f \in C_{0}^{\infty}(R)\right\}$ in $\Gamma$, where $C_{0}^{\infty}(R)$ is the totality of $C^{\infty}$-functions on $R$ with compact supports. We also denote by $* \Gamma_{e 0}=* \Gamma_{e 0}(R)=\left\{{ }^{*} \omega ; \omega \in \Gamma_{e 0}(R)\right\}$. Then we have the de Rahm decomposition of $\Gamma$ :

$$
\Gamma(R)=\Gamma_{e 0}(R) \oplus * \Gamma_{e 0}(R) \oplus \Gamma_{h}(R),
$$

Received by the editors August 16, 1965.

(1) This work was sponsored by the U.S. Army Research Office, Durham, Grant DAAROD-31-124-G499, University of California, Los Angeles. 
where $\Gamma_{h}=\Gamma_{h}(R)$ is the totality of harmonic differentials in $\Gamma(R)$. From these remarks, it follows at once that Problem $\mathrm{A}$ is equivalent to the following:

Problem B. Given a $C^{1}$-differential $\sigma$ on $R$ whose restriction to $\bar{R}_{1}$ is a harmonic singularity at $\beta$, find a harmonic differential $\lambda$ on $R$ such that $\lambda-\sigma \in \Gamma_{e 0}(R) \oplus * \Gamma_{e 0}(R)$.

The advantage of this reformulation is that we can now see the precise nature of the solution. That is, we shall show that if the solution $\lambda$ of Problem B exists, then it is unique and if $\sigma$ is exact (resp. *exact), then the solution $\lambda$ is also exact (resp. *exact). A differential is *exact, if, by definition, its *conjugate is exact (Theorem 2).

3. The key to the solution of our problem is the following: let $\mathscr{D}(\alpha)$ be the totality of continuous differentials $\omega$ defined on neighborhoods $V_{\omega}$ of $\alpha$ and $\mathscr{D}_{0}(\alpha)$ the subclass of $\mathscr{D}(\alpha)$ consisting of differentials $\omega$ such that $\int_{\alpha} \omega=0$. For each $\omega \in \mathscr{D}(\alpha)$, we consider the quantity

$$
K(\alpha, \omega)=\sup \left\{\left|\int_{\alpha} f \omega\right|^{2} / \int_{R} d f \wedge * d f ; f \in C_{0}^{\infty}(R), f \not \equiv 0\right\} .
$$

We shall show that if $R \notin O_{G}$, then $K(\alpha, \omega)<\infty$ for any $\omega \in \mathscr{D}(\alpha)$, and if $R \in O_{G}$, then $K(\alpha, \omega)<\infty$ if and only if $\omega \in \mathscr{D}_{0}(\alpha)$ (Theorem 1).

\section{Fundamental inequality}

4. Fix a point $z_{0}$ in $R_{0}$ and consider the space $H D_{0}=H D_{0}\left(R_{0}\right)$ of $H D$-functions on $R_{0}$ vanishing at $z_{0} . H D_{0}$ is a Hilbert space with respect to the norm $\int_{R_{0}} d u \wedge * d u$. For two fuilctions $u$ and $v$ in $H D_{0}$, we set

$$
(u, v)_{g}=\int_{R_{0}}\left(1+g\left(z, z_{0}\right)\right) d u(z) \wedge * d v(z)
$$

where $g$ is the Green's function on $R_{0} . H D_{0}$ is a pre-Hilbert space with this inner product, and we denote it by $H D_{0, g}$. Let $\left\{u_{n}\right\}_{n=1}^{\infty}$ be a Cauchy sequence in $H D_{0, g}$. Since it is also a Cauchy sequence in $H D_{0}$, there exists an element $u \in H D_{0}$ such that $d u_{n} \rightarrow d u$ in each parametric neighborhood. Then by Fatou's lemma

$$
\int_{R_{0}}\left(1+g\left(z, z_{0}\right)\right) d\left(u-u_{n}\right) \wedge * d\left(u-u_{n}\right) \leqq \liminf _{m \rightarrow \infty}\left(u_{m}-u_{n}, u_{m}-u_{n}\right)_{g}
$$

Thus we have that $u \in H D_{0, g}$ and

$$
\liminf _{n \rightarrow \infty}\left(u-u_{n}, u-u_{n}\right) \leqq \lim _{n, m \rightarrow \infty}\left(u_{m}-u_{n}, u_{m}-u_{n}\right)=0 .
$$

This shows that $H D_{0, g}$ is a Hilbert space. Clearly $u \rightarrow u$ is a continuous isomorphism of $H D_{0, g}$ onto $H D_{0}$. By the closed graph theorem, the norm $(u, u)_{g}$ is equivalent to $\int_{R_{0}} d u \wedge * d u$. Hence in particular, there is a constant $K_{1}$ such that

$$
\int_{R_{0}} g d u \wedge * d u \leqq K_{1} \int_{R_{0}} d u \wedge * d u
$$

for any $u$ in $H D_{0}$ and hence for any $u$ in $H D\left(R_{0}\right)$. 
Let $u \in H D_{0} \cap C\left(\bar{R}_{0}\right)$. Since $d^{*} d u^{2}=2 d u \wedge^{*} d u$, by Green's formula,

$$
\int_{\alpha} u^{2} * d g=2 \int_{R_{0}} g d u \wedge * d u
$$

Hence if $\omega \in \mathscr{D}_{0}(\alpha)$ and $u \in H D(R) \cap C\left(\bar{R}_{0}\right)$, then by (3), (4), and the fact $\int_{\alpha} u \omega$ $=\int\left(u-u\left(z_{0}\right)\right) \omega$, we deduce

$$
\left|\int_{\alpha} u \omega\right|^{2} \leqq K_{2} \int_{R_{0}} d u \wedge * d u
$$

where $K_{2}=2 K_{1} \int_{\alpha} \Omega^{2} * d g$ with $\Omega * d g=\omega$ on $\alpha$. Hence by using Dirichlet's principle, we obtain the following:

Lemma 1 [3]. Let $\omega \in \mathscr{D}_{0}(\alpha)$. Then there exists a constant $C_{\omega}$ depending only on $\omega$ such that for any $f \in C^{1}\left(R_{0}\right) \cap C\left(\bar{R}_{0}\right)$,

$$
\left|\int_{\alpha} f \omega\right|^{2} \leqq C_{\omega} \int_{R_{0}} d f \wedge * d f
$$

5. Assume that $R \notin O_{G} . R_{1}=R-\bar{R}_{0}$ consists of a finite number of components $R_{1}^{(1)}, R_{1}^{(2)}, \ldots, R_{1}^{(k)}$. Let $\alpha_{i}=\alpha \cap \partial R_{1}^{(i)}$. If $R_{1}^{(i)}$ has positive (resp. null) ideal boundary, then we put $F_{i}=R_{1}^{(i)}\left(\right.$ resp. $\left.F_{i}=R-\left(R_{1}^{(i)}\right)^{-}\right)$and orient $\alpha_{i}=\partial F_{i}$ positively with respect to $F_{i}$. Take the harmonic measure $w_{i}$ on $F_{i}$ such that $w_{i}=0$ on $\partial F_{i}$. Since $R \notin O_{G}$, at least one of $R_{1}^{(i)}$ and $R-\left(R_{1}^{(i)}\right)^{-}$has positive ideal boundary and by our choice of $F_{i}$, $w_{i}>0$. Take an $f$ in $C_{0}^{\infty}(R)$ arbitrary but fixed for the time being. Let $F_{i}^{\prime}$ be a subdomain of $F_{i}$ such that $F_{i}-\left(F_{i}^{\prime}\right)^{-}$is a neighborhood of the ideal boundary of $F_{i}$ and $f$ vanishes on $F_{i}-F_{i}^{\prime}$ and such that $\partial F_{i}^{\prime}$ consists of a finite number of mutually disjoint analytic closed Jordan curves with $\alpha_{i} \subset \partial F_{i}^{\prime}$. We orient $\beta_{i}=\partial F_{i}^{\prime}-\alpha_{i}$ positively with respect to $F_{i}^{\prime}$. Let $u$ be harmonic in $F_{i}^{\prime}$ with boundary values $f$ on $\partial F_{i}^{\prime}$. Hence $u=f$ on $\alpha_{i}$ and $u=0$ on $\beta_{i}$. By Green's formula

$$
\int_{\alpha_{i}+\beta_{i}} u^{2} * d w_{i}-\int_{\alpha_{i}+\beta_{i}} w_{i} * d u^{2}=\int_{F_{i}} w_{i} d * d u^{2}
$$

Since $* d u^{2}=2 u^{*} d u=0$ on $\beta_{i}$ and $d^{*} d u^{2}=2 d u \wedge * d u$, we have

$$
\int_{\alpha_{i}} u^{2 *} d w_{i}=2 \int_{r_{i}^{\prime}} w_{i} d u \wedge * d u \text {. }
$$

Hence by noticing $w_{i}<1$,

$$
\left|\int_{\alpha_{i}} u \omega\right|^{2} \leqq K_{\omega}^{(i)} \int_{r_{i}^{i}} d u \wedge * d u,
$$

where $\omega \in \mathscr{D}(\alpha)$ and $K_{\omega}^{(i)}=2 \int_{\alpha_{i}} \Omega^{2 *} d w_{i}$ with $\Omega * d w_{i}=\omega$ on $\alpha$. Thus by $\int_{F_{i}^{i}} d u$ $\wedge^{*} d u \leqq \int_{F_{i}} d f \wedge^{*} d f \leqq \int_{R} d f \wedge^{*} d f$ and $u=f$ on $\alpha_{i}$, we have

$$
\left|\int_{\alpha_{i}} f \omega\right|^{2} \leqq K_{\omega}^{(i)} \int_{R} d f \wedge * d f
$$


Let $K_{\omega}=k \max \left(K_{\omega}^{(i)} ; 1 \leqq i \leqq k\right)$. Then

$$
\left|\int_{\alpha} f \omega\right| \leqq \sum_{i=1}^{k}\left|\int_{\alpha_{i}} f \omega\right| \leqq \sqrt{K_{\omega} \int_{R} d f \wedge * d f}
$$

Hence we obtain the following:

Lemma 2. Let $R \notin O_{G}$ and $\omega \in \mathscr{D}(\alpha)$. Then there exists a constant $K_{\omega}$ depending only on $\omega$ such that for any $f \in C_{0}^{1}(R)$,

$$
\left|\int_{\alpha} f \omega\right|^{2} \leqq K_{\omega} \int_{R} d f \wedge * d f
$$

6. Assume that $R \in O_{G}$. Let $\omega \in \mathscr{D}(\alpha)$ and assume that there exists a constant $C_{\omega}$ depending only on $\omega$ such that

$$
\left|\int_{\alpha} f \omega\right|^{2} \leqq C_{\omega} \int_{R} d f \wedge * d f
$$

for any $f \in C_{0}^{\infty}(R)$. Let $\Omega$ be a subdomain of $R$ such that $\Omega \supset \bar{R}_{0}$ and $\partial \Omega$ consists of a finite number of mutually disjoint analytic closed Jordan curves. Let $w_{\Omega}$ be the continuous function on $R$ such that $w_{\Omega}=1$ on $\bar{R}_{0}, w_{\Omega}=0$ on $R-\Omega$ and $w_{\Omega}$ is harmonic on $\Omega-\bar{R}_{0}$. By applying the mollifier, we can find a sequence $\left\{f_{n}\right\}$ in $C_{0}^{\infty}(R)$ such that $\int_{R} d\left(w_{\Omega}-f_{n}\right) \wedge * d\left(w_{\Omega}-f_{n}\right) \rightarrow 0(n \rightarrow \infty)$ and $f_{n}$ converges uniformly to $w_{\Omega}$ on $R$. Then from

$$
\begin{gathered}
\left|\int_{\alpha} f_{n} \omega\right|^{2} \leqq C_{\omega} \int_{R} d f_{n} \wedge * d f_{n} \\
\left|\int_{\alpha}\left(f_{n}-1\right) \omega\right| \leqq\left(\int_{\alpha}|\omega|\right) \sup \left|f_{n}-w_{\Omega}\right|
\end{gathered}
$$

it follows that

$$
\left|\int_{\alpha} \omega\right|^{2} \leqq C_{\omega} \int_{\Omega-\Sigma_{0}} d w_{\Omega} \wedge * d w_{\Omega} .
$$

Since $R \in O_{G}, \int_{\Omega-\bar{R}_{0}} d w_{\Omega} \wedge^{*} d w_{\Omega} \rightarrow 0$ as $\Omega \nearrow R$. Thus $\int_{\alpha} \omega=0$. Hence this with Lemma 1 gives the following:

Lemma 3. Let $R \in O_{G}$ and $\omega \in \mathscr{D}(\alpha)$. In order that there exists a constant $C_{\omega}$ depending only on $\omega$ such that for any $f \in C_{0}^{1}(R)$,

$$
\left|\int_{\alpha} f \omega\right|^{2} \leqq C_{\omega} \int_{R} d f \wedge * d f
$$

it is necessary and sufficient that $\omega \in \mathscr{D}_{0}(\alpha)$.

7. Lemmas 2 and 3 complete the proof of the fact mentioned in 3:

Theorem 1. If $R \notin O_{G}$, then $K(\alpha, \omega)<\infty$ for any $\omega \in \mathscr{D}(\alpha)$, while if $R \in O_{G}$, then $K(\alpha, \omega)<\infty$ if and only if $\omega \in \mathscr{D}_{0}(\alpha)$. 


\section{Existence theorem}

8. THEOREM 2. Let $\sigma$ be a $C^{1}$-differential on $R$ such that $\sigma \mid \bar{R}_{1}$ gives a harmonic singularity at the ideal boundary $\beta$ of $R$. If $R \notin O_{G}$, then there exists a harmonic differential $\lambda$ on $R$ such that $\lambda-\sigma \in \Gamma_{e 0}(R) \oplus{ }^{*} \Gamma_{e 0}(R)$, and if $R \in O_{G}$, then there exists a harmonic differential $\lambda$ on $R$ such that $\lambda-\sigma \in \Gamma_{e 0}(R) \oplus{ }^{*} \Gamma_{e 0}(R)$ if and only if

$$
\int_{\alpha} \sigma=\int_{\alpha} *_{\sigma}=0 .
$$

In either case $\lambda$ is uniquely determined by $\sigma$ and $\lambda$ is exact (resp. ${ }^{*}$ exact) if $\sigma$ is exact (resp. ${ }^{*}$ exact).

9. First, we prove the existence part. Let $\sigma$ be arbitrary if $R \notin O_{G}$ and let $\sigma$ satisfy (10) if $R \in O_{G}$. Let $f \in C_{0}^{\infty}(R)$. By Green's formula,

$$
\int_{R_{1}} \sigma \wedge * d f=\int_{R_{1}} d f \wedge *_{\sigma}=\int_{\alpha} f^{*} \sigma
$$

and

$$
\int_{R_{1}} \sigma \wedge d f=-\int_{R_{1}} d f \wedge \sigma=-\int_{\alpha} f \sigma .
$$

Hence for any $f_{1}$ and $f_{2}$ in $C_{0}^{\infty}(R)$, by Theorem 1 and $\left(d f_{1},{ }^{*} d f_{2}\right)=0$,

$$
\left|\int_{R_{1}} \sigma \wedge *\left(d f_{1}+* d f_{2}\right)\right|^{2} \leqq T_{\sigma}^{\prime} \int\left(d f_{1}+* d f_{2}\right) \wedge *\left(d f_{1}+* d f_{2}\right),
$$

where $T_{\sigma}^{\prime}$ is a constant depending only on $\sigma$. Thus the functional

$$
T(\theta)=-\int_{R} \sigma \wedge * \theta
$$

defined on $\Gamma_{e 0}^{\infty}(R) \oplus * \Gamma_{e 0}^{\infty}(R)$ satisfies

$$
|T(\theta)|^{2} \leqq T_{\sigma} \int_{R} \theta \wedge * \theta,
$$

where $T_{\sigma}=\int_{R_{0}} \sigma \wedge *^{*} \sigma+T_{\sigma}^{\prime}$. Thus $T$ can be extended to a bounded linear functional on

$$
\Gamma_{e 0}(R) \oplus * \Gamma_{e 0}(R)=\overline{\Gamma_{e 0}^{\infty}(R) \oplus \oplus^{*} \Gamma_{e 0}^{\infty}(R)} .
$$

Hence there exists a unique element $\omega$ in $\Gamma_{e 0}(R) \oplus{ }^{*} \Gamma_{e 0}(R)$ such that $T(\theta)=\int \omega \wedge * \theta$. Thus in particular,

$$
\int_{R}(\sigma+\omega) \wedge * d f=\int_{R}(\sigma+\omega) \wedge d f=0
$$

for any $f$ in $C_{0}^{\infty}(R)$. Take a compact subdomain $\Omega$ in $R$; then clearly $\sigma+\omega \in \Gamma(\Omega)$. Taking $f$ in $C_{0}^{\infty}(\Omega)$, the above equality shows that $\sigma+\omega \in\left(\Gamma_{e 0}^{\infty}(\Omega) \oplus{ }^{*} \Gamma_{e 0}^{\infty}(\Omega)\right)^{\perp}$. By the de Rahm decomposition, $\Gamma_{h}(\Omega)=\Gamma(\Omega) \ominus\left(\Gamma_{e 0}^{\infty}(\Omega) \oplus{ }^{*} \Gamma_{e 0}(\Omega)\right)$, we conclude 
that $\sigma+\omega \in \Gamma_{h}(\Omega)$. Thus $\lambda=\sigma+\omega$ is a harmonic differential on $R$ and $\lambda-\sigma$ $=\omega \in \Gamma_{e 0}(R) \oplus * \Gamma_{e 0}(R)$.

10. Next assume that $R \in O_{G}$ and we can find a harmonic differential $\lambda$ on $R$ such that $\lambda-\sigma=\omega \in \Gamma_{e 0}(R) \oplus{ }^{*} \Gamma_{e 0}(R)$ for a given $\sigma$. We have to show that (10) holds. Clearly, for any $f \in C_{0}^{\infty}(R)$,

$$
\left|\int_{\alpha} f \omega\right|^{2}=\left|\int_{R_{1}} d f \wedge \omega\right|^{2} \leqq\left(\int_{R} \omega \wedge * \omega\right) \cdot \int_{R} d f \wedge * d f
$$

and similarly

$$
\left|\int_{\alpha} f^{*} \omega\right|^{2} \leqq\left(\int_{R} \omega \wedge^{*} \omega\right) \cdot \int_{R} d f \wedge * d f
$$

Hence by Theorem 1 , we must have $\int_{\alpha} \omega=\int_{\alpha}{ }^{*} \omega=0$. On the other hand, $\int_{\alpha} \lambda$ $=\int_{R_{0}} d \lambda=0$ and $\int_{\alpha} * \lambda=\int_{R_{0}} d^{*} \lambda=0$. Thus (10) follows.

11. Finally, we prove the last part of Theorem 2. Let $\lambda_{1}$ and $\lambda_{2}$ be harmonic differential on $R$ such that $\lambda_{1}-\sigma$ and $\lambda_{2}-\sigma$ belong to $\Gamma_{e 0}(R) \oplus *\left(\Gamma_{e 0}(R)\right)$. Then $\lambda_{1}-\lambda_{2} \in \Gamma_{e 0}(R) \oplus * \Gamma_{e 0}(R)$. But $\lambda_{1}-\lambda_{2}$ is harmonic and thus $\lambda_{1}-\lambda_{2}=0$ by the de Rahm decomposition.

Assume that $\sigma$ is exact (resp. *exact). Then for $\theta \in * \Gamma_{e 0}^{\infty}(R)\left(\operatorname{resp} . \Gamma_{e 0}^{\infty}(R)\right)$, $\int_{R} \lambda \wedge * \theta=\int_{R} \sigma \wedge * \theta=0$. This implies $\int_{R} \omega \wedge^{*} \theta=0$. Thus $\omega \in\left(\Gamma_{e 0}(R) \oplus * \Gamma_{e 0}(R)\right)$ $\ominus^{*} \Gamma_{e 0}^{\infty}(R)=\Gamma_{e 0}(R)$ (resp. $\omega \in\left(\Gamma_{e 0}(R) \oplus * \Gamma_{e 0}(R)\right) \ominus \Gamma_{e 0}^{\infty}(R)=* \Gamma_{e 0}(R)$ ).

12. Application to the case of functions. Let $s$ be a harmonic function on $\bar{R}_{1}$, arbitrary if $R \notin O_{G}$, and $\int_{\alpha}{ }^{*} d s=0$ if $R \in O_{G}$. Thus by Theorem 2 , there exists a harmonic function $p$ on $R$ such that $d(p-s) \in \Gamma_{e 0}(R)$. This means that $p-s$ is bounded on $R$. Thus we constructed a harmonic function $p$ on $R$ which behaves like $s$ at $\beta$. If $\int_{\alpha} * d s=0$, then $\int_{\alpha} * d(p-s)=0$ and $d(p-s) \in \Gamma_{e 0}(R)$. This implies $L_{1}(p-s)=p-s$ on $R_{1}$ (see [3]), where $L_{1}$ is Sario's principal operator for $R_{1}$. Such an approach to the principal function problem was initiated by Browder [2].

The author wishes to express his gratitude to Professor Leo Sario for his stimulating suggestions.

\title{
REFERENCES
}

1. L. V. Ahlfors and L. Sario, Riemann surfaces, Princeton Math. Series, No. 26, Princeton, N. J., 1960.

2. F. E. Browder, Principal functions for elliptic systems of differential equations, Bull. Amer. Math. Soc. 71 (1965), 342-344.

3. M. Nakai and L. Sario, Construction of principal functions by orthogonal projections, Canad. J. Math. 18 (1966), 887-896.

\author{
UNIVERSITY OF CALIFORNIA, \\ Los Angeles, California \\ Nagoya University, \\ NAGOYA, JAPAN
}

